Universidad de Lima

Facultad de Psicología

Carrera de Psicología

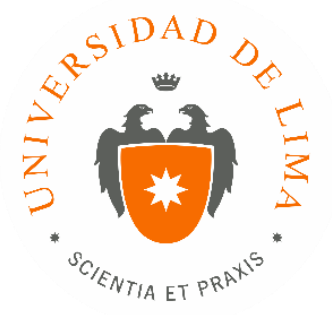

\title{
LOS BENEFICIOS DEL SALARIO EMOCIONAL PARA LA ORGANIZACIÓN Y LOS TRABAJADORES
}

Trabajo de suficiencia profesional para optar el título profesional de Licenciado en Psicología

\section{Verónica Zapata Pflucker}

Código 20112503

$$
\text { Lima - Perú }
$$

Febrero de 2019 


\section{LOS BENEFICIOS DEL SALARIO EMOCIONAL PARA LA ORGANIZACIÓN Y LOS TRABAJADORES}




\section{TABLA DE CONTENIDO}

INTRODUCCIÓN

CAPÍTULO I: IDENTIFICACIÓN DEL PROBLEMA ...............................................

CAPÍTULO II: DESCRIPCIÓN DE LAS ACTIVIDADES REALIZADAS .................. 10

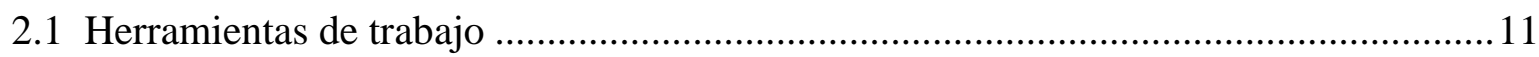

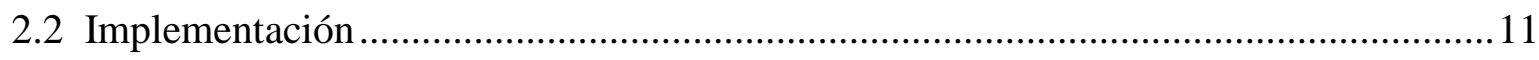

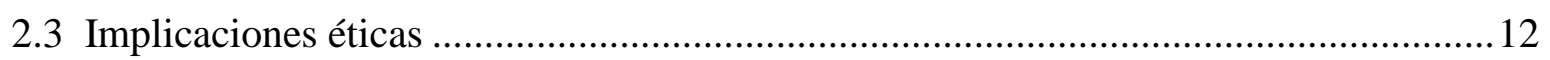

CAPÍTULO III: RESULTADOS DE LA INTERVENCIÓN........................................ 14

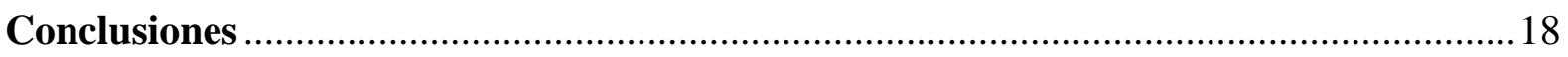

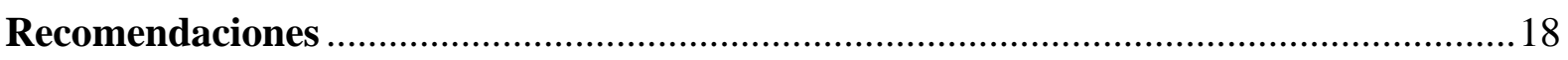

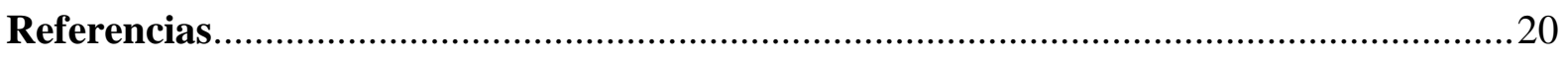

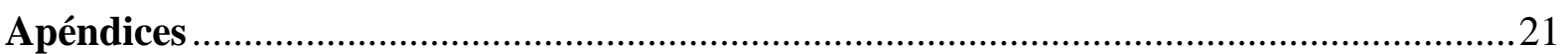




\section{ÍNDICE DE TABLAS}

Tabla 3.1: Indicadores de Productividad por cierre de procesos .16

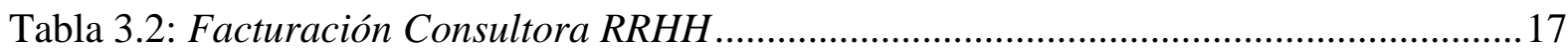

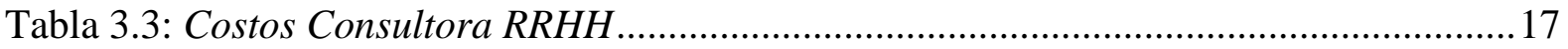




\section{ÍNDICE DE APÉNDICES}

Apéndice 1: Cuestionario de Salario Emocional .....

Apéndice 2: Guía de Entrevista. 


\section{INTRODUCCIÓN}

Muchos de los profesionales reclutadores y algunas empresas consideran que para atraer y retener personal es únicamente importante la oferta económica y los beneficios adicionales a los de ley que suelen darse como la alimentación y el seguro médico privado contratado por la empresa también conocido como EPS. Las ofertas que solo se componen por estos beneficios no son competitivas y por ende se restringe la calidad del personal a reclutarse.

Virna Vitteri (2017), señala que hay distintos motivos por los que las personas pueden preferir trabajar en una empresa u otra, pero sí puede decirse que la mayoría de elementos tienen que ver con el ambiente laboral, los cuales pueden afectar la motivación de los profesionales de una empresa.

Sin embargo, existen más aspectos que deben ser considerados en la propuesta de valor al candidato y/o trabajador de la empresa. La ausencia de estos, pueden motivar al personal a buscar nuevas alternativas para cubrir sus necesidades y a su vez la empresa verse afectada por la escasez de talento, impactando en su productividad, rentabilidad y marca empleadora. A estos otros aspectos se les conoce como salario emocional. Se suele tomar en cuenta el salario emocional cuando la corporación no tiene la solvencia económica para aumentar los sueldos, ya que este tipo de beneficios es más rentable.

Aparicio (2017) se refiere al salario emocional como una motivación para que el personal se desarrolle de forma más productiva, logrando aumentar el rendimiento, tener mayor proactividad, mayor compromiso con los objetivos de la empresa y fomentar el sentido de pertenencia.

Es por ello que en este trabajo, se podrá encontrar cómo la implementación del salario emocional en una consultora de recursos humanos, que cuenta con más de 20 años en el mercado, generó un impacto positivo en la empresa y sus colaboradores. 


\section{CAPÍTULO I: IDENTIFICACIÓN DEL PROBLEMA}

A lo largo de la carrera de psicología se ha aprendido que un psicólogo empresarial busca el bienestar del trabajador y de la empresa, el cual debe ser medido mediante indicadores para mejorar la objetividad y reducir la subjetividad. Deloitte (2018), reconoce que una gestión estratégica de Recursos Humanos es reconocer que el capital más valioso de las organizaciones son los empleados y que son un activo fundamental para la el logro de los objetivos.

Es por ello que en esta ocasión se hizo un diagnóstico dentro de una consultora de recursos humanos. A través de entrevistas y encuestas a 14 de los colaboradores de la empresa entre contratados y practicantes, se logra identificar que los trabajadores no se encontraban totalmente satisfechos con su remuneración y requerían un aumento salarial, pero para la empresa no era viable en ese momento, ya que se estaban recuperando de una crisis financiera, debido a que los cambios políticos y económicos del país impactaron negativamente en el negocio. Asimismo, se aprecia que los colaboradores se encuentran disconformes con los beneficios actuales que son: EPS al $100 \%$, horario de verano todos los viernes del año y permisos regulados, este descontento se debe a que ya se encontraban acostumbrados a los mismos y además percibían un ambiente poco flexible, que los limitaba a mantener un equilibrio personal, laboral e incluso poder continuar con su desarrollo profesional. Por otro lado, se identificó que los colaboradores no estaban dando el $100 \%$ de su potencial lo cual se veía reflejado en los indicadores del negocio y comentarios de algunos de los clientes.

Según la Consultora Mercer (2018), en su estudio sobre tendencias globales de talento, el 71\% de los empleados prósperos señalan que su compañía les ofrece modalidades de trabajo flexible, en comparación con el 32\% de los individuos que no logran prosperar en su trabajo. 
Teniendo en cuenta el diagnóstico realizado y que una de las principales funciones de un psicólogo organizacional es que los procesos de Recursos Humanos se encuentren orientados al personal con la finalidad de mejorar el servicio para que de esta manera ellos se logren enfocar en la productividad, se planteó la posibilidad de implementar el concepto de salario emocional dentro de la organización ante la falta de recursos económicos de la empresa para aumentar los sueldos de los empleados y ante la percepción de los mismos sobre la falta de beneficios y flexibilidad.

Según la Asociación Española para la Calidad (2013), el concepto de salario emocional se encuentra asociado a la retribución no económica, que tiene la finalidad de satisfacer las necesidades personales, familiares y profesionales de los empleados, proporcionándoles una mejor calidad de vida. 


\section{CAPÍTULO II: DESCRIPCIÓN DE LAS ACTIVIDADES Y TAREAS REALIZADAS}

Para poder iniciar la incorporación del concepto de salario emocional dentro de la organización es importante tener en claro su definición e implicaciones. Es por ello que se planteó una definición general tomando en cuenta distintas fuentes de referencia. Se llegó a la conclusión que el salario emocional se refiere a todas las retribuciones no económicas que el colaborador puede obtener como beneficio en la organización con el objetivo de incentivar de forma positiva la imagen que tiene el colaborador sobre su ambiente de trabajo, lograr incrementar su productividad y satisfacer sus necesidades personales, familiares y profesionales.

Sánchez (2014), manifiesta que el salario emocional son los incentivos no monetarios, por el cual se reconocen los logros que alcanzan los trabajadores, con la finalidad de cubrir sus necesidades. Tomando en cuenta los tipos de retribución intrínseca; afiliación, tomar en cuenta opiniones, crecimiento profesional, crecimiento personal y extrínseca; políticas de viaje, flexibilidad de horarios, parking, entre otros.

Asimismo, se toman distintos ejemplos sobre salario emocional para poder realizar las entrevistas y encuestas. Dentro de estas se encuentra el horario flexible, el cual significa cumplir el horario establecido enfocado en objetivos, pero sin estar las 8 horas en la oficina. También se tomó en cuenta el trabajo de home office o teletrabajo que permite al colaborador trabajar de forma remota, la guardería para aquellos que son padres de familia, días libres por eventos especiales, espacios de distracción o recreativos dentro de la oficina, capacitaciones reconocimientos, entre otros.

Según Conrado Castillo (2009), son cuatro los aspectos que las empresas deben tener en cuenta para poder retener talento: Factores de desarrollo profesional y relacional con superiores o compañeros, factores de compensación psicológica; que involucra el sentido de pertenencia, 
autonomía, utilidad y reconocimiento, factores de conciliación personal y familiar, tanto en aspectos económicos como domésticos y factores de retribución variable y fija, que involucra la participación activa de los colaboradores en los resultados de la empresa.

Por otro lado, se debe tomar en cuenta que el salario emocional ha sido efectivo en organizaciones extranjeras y peruanas. Actualmente en Perú se está implementando poco a poco sobretodo en grandes organizaciones obteniendo resultados satisfactorios. Es por ello que se planteó realizar este proyecto de implementación, ya que marcaría un diferencial tanto de cara al cliente como para la atracción y retención del talento.

Valls en su libro "Big Data: atrapando al consumidor" (2017), señala que los aspectos por los que está conformado el salario emocional varían en base a las generaciones, pues cada generación e individuo tiene un concepto distinto de sus necesidades, capacidades, motivaciones, aspiraciones. Llegando a la conclusión que los millennials se interesan por ser reconocidos, reclaman liderazgo, plan de carrera; es decir, inclinándose hacia el salario emocional, mientras que los demás prefieren las retribuciones y bonificaciones económicas.

\subsection{Herramientas de trabajo}

En el presente trabajo se utilizaron dos herramientas principales para levantar información: la entrevista y un cuestionario de salario emocional, diseñado por el equipo de implementación. A través de la entrevista, se identificaron criterios generales de los entrevistados como tiempo de pertenencia en la empresa, beneficios actuales, diferencias entre la consultora y otras empresas y satisfacción laboral, en donde se evidencia que los trabajadores no se encontraban conformes con sus sueldos y que la mayoría se hallaban en la búsqueda activa de nuevas ofertas laborales con la finalidad de satisfacer sus necesidades económicas y no económicas, ya que la consultora no llegaba a satisfacerlas en su totalidad. Por último, se indagó 
acerca del conocimiento de los empleados sobre el concepto de salario emocional, para tomarlo como referencia para el proyecto. Por otro lado, el cuestionario permitió evaluar cuáles son los aspectos que a los empleados les gusta de la organización y cuáles son las necesidades más importantes que deben abarcarse dentro del salario emocional, para generar un impacto positivo deseado en la satisfacción del personal y productividad. Se evidencia que el empleador proporciona regularmente normativas a seguir y planes de entrega de servicios que favorecen su crecimiento personal y profesional, les ofrece un espacio y ambiente que les facilita realizar su trabajo. Asimismo, consideran que la empresa les permite explotar su potencial y que al recibir "feedback" de sus compañeros y jefes les facilita desarrollarse. Sin embargo, los trabajadores se encuentran insatisfechos con su remuneración, perciben que sus opiniones y comentarios no son valorados o tomados en cuenta, no se les brinda flexibilidad para trabajar desde casa, para asistir a citas médicas y la mayoría señala que presentan dificultades para asistir a eventos del colegio de sus hijos. Igualmente, se determina que el personal de la consultora no se encuentra satisfecho con las capacitaciones y el reconocimiento que la empresa les otorga.

\subsection{Implementación}

En primer lugar, se presentó el concepto de salario emocional a los trabajadores de la empresa, tomando en cuenta los conceptos que ellos señalaban como salario emocional y la bibliografía revisada. Por otro lado, se identificó que la población a la que va dirigida el proyecto está compuesta por 14 trabajadores, siendo 4 coordinadoras, 6 practicantes, 2 consultores y 2 personas dedicadas a realizar labores administrativas. Del total de la población 3 son hombres y 11 son mujeres y 
las edades se encuentran en un rango de 23 a 40, por lo que se deduce que las necesidades son diversas.

En segundo lugar, en base a las necesidades levantadas se tomaron en cuenta las 5 más relevantes y se puso en práctica la implementación de distintos beneficios para cumplir con la satisfacción de las mismas. Dentro de las principales se encontraron la necesidad de los colaboradores por asistir a los distintos eventos que realizaban los colegios de sus hijos, asistir a citas médicas que no pueden ser programadas fuera de horario laboral, tener flexibilidad de horario que les permita estudiar o seguir capacitándose, tener la posibilidad de trabajar desde sus casas lo cual según indican les permite mantener un equilibrio en la vida personal- laboral y de que se tomen en cuenta sus opiniones, sugerencias y propuestas. Tomando en cuentas estas necesidades se propuso que durante un periodo de 8 meses los colaboradores tendrían la posibilidad de tener home office dos veces al mes, medio día libre una vez al mes, se propuso que el primer lunes de cada mes, los colaboradores tendrían una reunión para brindar opiniones, propuestas o conversar sobre algo en particular, de igual manera se propuso mantener una comunicación horizontal y constante, a través de un grupo de chat tanto en WhatsApp como en Gmail, y por último, se brindó flexibilidad de horario para los trabajadores que opten por seguir estudiando. Si bien la implementación de este proyecto no involucro un desembolso de dinero en el flujo de caja que se viene dando, tiene el costo de las horas hombre en las que los trabajadores dejarían de laboral en el caso del medio día libre y la flexibilidad de horarios por motivos académicos; sin embargo, este costo se compensa con la mejora en la productividad y rentabilidad, cálculos que se mostrarán en los resultados. 


\subsection{Implicaciones éticas}

Es importante tomar en cuenta las implicaciones éticas dentro del desarrollo del proyecto y el trabajo. En primer lugar, se debió mantener la confidencialidad en toda la información levantada y se mantiene los comentarios, entrevistas y cuestionarios de los empleados bajo seudónimos con la finalidad de evitar un mal clima laboral. Asimismo, se tomó en cuenta dentro del presente trabajo que por principios éticos no se puede publicar el nombre de la organización ni tampoco anexar las transcripciones de las entrevistas, cuestionario resuelto, etc. 


\section{CAPÍTULO III: RESULTADOS DE LA INTERVENCIÓN}

En términos generales, la implementación del salario emocional dentro de la organización generó un impacto positivo. Se logró reducir la rotación, fidelizando a los trabajadores logrando satisfacer sus necesidades no económicas, generando también un mejor clima laboral y evitando que los colaboradores se encuentren en la búsqueda de otras oportunidades laborales. Asimismo, se logró que las practicantes se queden no solo el tiempo de prácticas pre profesionales sino también hagan prácticas profesionales hasta que se aperture la posibilidad de un nuevo "headcount". Se pudo evidenciar que este proyecto permitió que el personal logre mayores niveles de productividad, el cual se ve reflejado en los indicadores. Por otro lado, se evidencia una mayor satisfacción por parte de los clientes actuales externos, lo cual se refleja en la retroalimentación recibida y en las recomendaciones de los mismos que permitieron que la empresa tenga nuevos clientes.

En primer lugar, como se mencionó, se logró fidelizar a los trabajadores, se incrementó su sentido de pertenencia y mejoró el nivel de satisfacción laboral, evitando que los colaboradores busquen otras ofertas labores, disminuyendo a su vez la rotación de personal en especial de parte de los practicantes quienes son lo que más buscan nuevas oportunidades de trabajo, en base a la información levantada. Esto se pudo medir mediante las entrevistas realizadas a los colaboradores. Del total de los 16 trabajadores entre empleados y practicantes se entrevistó a 14, dejando fuera de la entrevista al gerente general y a quien realizó el proyecto, para evitar sesgos y obtener resultados más confiables. Antes de la implementación, el $57.1 \%$ de los entrevistados mencionaron que se encontraban en la búsqueda activa de trabajo, debido a que la empresa no satisfacía sus necesidades tanto económicas como no económicas, buscaban una organización que les permita mantener un equilibrio personal y profesional, y poder actualizarse y desarrollarse a nivel académico. Por otro lado, el $42.9 \%$ se sentía satisfecho con 
los beneficios que la empresa ofrecía en especial con la posibilidad de salir los viernes a la 1:00, lo cual les permitía realizar distintas actividades personales que en un horario normal no podrían realizar. Al terminar la implementación de los nuevos beneficios, basados en el concepto de salario emocional se logró evidenciar que el $28.6 \%$ de los trabajadores se encontraban en búsqueda de trabajo mientras que el $71.4 \%$ señala que el cambio que tuvo la organización les permitió sentirse más cómodos y que los beneficios les permiten sentirse más estables y les brindan una mejor calidad de vida personal, así como incrementar su productividad, desarrollándose profesionalmente.

Por otro lado, se logró un aumento en el nivel de productividad de los colaboradores dedicados al reclutamiento de personal, evidenciado en los indicadores de cierre de procesos de selección, el cual se puede comprobar en la siguiente tabla:

Tabla 3.1

Indicadores de Productividad por cierre de procesos

\begin{tabular}{cccc}
\hline Equipo & Trabajador & Antes & Después \\
\hline Equipo 1 & A.O & $50 \%$ & $90 \%$ \\
Equipo 1 & V.Z & $80 \%$ & $100 \%$ \\
Equipo 1 & D.R & $40 \%$ & $60 \%$ \\
Equipo 2 & M.V. & $60 \%$ & $90 \%$ \\
Equipo 2 & M.B & $55.60 \%$ & $77.80 \%$ \\
Equipo 2 & T.B & $60 \%$ & $80 \%$ \\
Equipo 3 & F.V & $70 \%$ & $80 \%$ \\
Equipo 3 & R.S & $66.70 \%$ & $88.90 \%$ \\
Equipo 3 & V.G & $80 \%$ & $80 \%$ \\
Equipo 4 & P.C & $80 \%$ & $90 \%$ \\
Equipo 4 & G. B & $60 \%$ & $60 \%$ \\
Equipo 4 & LC & $40 \%$ & $60 \%$ \\
\hline
\end{tabular}


Asimismo, se pudo evidenciar que los clientes se encontraban satisfechos con el trabajo realizado y también con la mejora de atención de parte del personal administrativo quien generaba las facturas con mayor rapidez, dentro de los plazos de tiempo establecidos y les brindaba un trato cordial y personalizado. Esto se evidenció con comentarios y con recomendaciones, que fueron evidenciadas con el aumento de clientes. Se pasó de tener un total de 16 cliente a tener 23 clientes, de los cuales, 5 fueron por recomendaciones de nuestros clientes, mientras que los otros 2 fueron adquiridos por la gerencia de la consultora.

Finalmente, el impacto de estas actividades generó una rentabilidad del 83\% en función a los cálculos detallados a continuación.

Tabla 3.2

Facturación Consultora RRHH

\begin{tabular}{cccc}
\hline Factores & Antes de la Implementación & $\begin{array}{c}\text { Después de la } \\
\text { Implementación }\end{array}$ & Incremento \\
\hline $\begin{array}{c}\text { Facturación Total (S/. sin } \\
\text { IGV) } \\
\text { Total de Clientes }\end{array}$ & 75,000 & 82,500 & 7,500 \\
\hline
\end{tabular}

Tabla 3.3

Costos Consultora RRHH

\begin{tabular}{lccc}
\hline Factores & Antes de la Implementación & $\begin{array}{c}\text { Después de la } \\
\text { Implementación }\end{array}$ & Reducción \\
\hline $\begin{array}{l}\text { Número de horas hombre } \\
\text { trabajadas por total de } \\
\text { trabajadores al mes }\end{array}$ & 2,352 & 2,286 \\
Costo Mano de Obra por & 18.88 & 1246.08 \\
Hora (S/.) & & \\
\hline Los costos no implican flujo de dinero adicional para la empresa.
\end{tabular}

Los costos no implican flujo de dinero adicional para la empresa. 


\section{CONCLUSIONES}

A continuación se detallan las conclusiones:

- El constante cambio en el ritmo de trabajo, las demandas de los clientes y la exigencia del empleador son factores clave que afectan a los colaboradores y muchas veces generan estrés es por eso que para hacer frente a este riesgo, una buena herramienta es el salario emocional, ya que contribuye a la salud de los empleados, además de mejorar la productividad e incrementar la innovación.

- El salario emocional impulsa al personal al logro de metas tanto corporativas como personales porque se busca resultados, reconocimientos y autorrealización, impactando de forma positiva en la satisfacción laboral, rendimiento, productividad y desarrollo profesional.

- Por los resultados se determina que las empresas al tener una alta variedad de trabajadores con características distintas cuentan con una gran cantidad de necesidades incluso distintas, logrando evidenciar que dentro de las principales se encuentran el equilibrio de la vida personal- laboral, flexibilidad de horarios y que la empresa brinde facilidades para continuar el desarrollo profesional.

- Se deduce que tanto los trabajadores como los practicantes buscan el salario emocional dentro de los requisitos indispensables para postular a las organizaciones, ya que es considerado un valor agregado para la misma. 


\section{RECOMENDACIONES}

A continuación se detallaran las recomendaciones:

- Se recomienda tener en cuenta el salario emocional como una posible solución para el estrés del colaborador ante las exigencias del entorno, ya que permitirá que el personal perciba que la organización entiende sus necesidades y a su vez satisfacerlas y de esta manera generar un mejor clima y reducir el estrés.

- Se considera recomendable la implementación del beneficio de salario emocional en las organizaciones, debido a que genera beneficios tanto para la empresa como para el trabajador, logrando la mejor productividad, alcanzando altos niveles de calidad en el trabajo de los empleados, satisfacción laboral y sentido de pertenencia.

- Tomando en cuenta que las organizaciones cuentan con una amplia cantidad de trabajadores, se recomienda que se evalúen, organicen y busquen satisfacer las necesidades de los mismos en base a su perfil, ya que cada individuo presenta demandas distintas, sin tener que implementar una solución por cada una.

- Se recomienda que para retener y atraer talento se tenga dentro de sus beneficios el salario emocional. Ya que es lo que actualmente están buscando los profesionales para poder mantener un equilibrio en sus vidas personales y profesionales. 


\section{REFERENCIAS}

Aparicio, P. S. (2017). Trabaja Inteligente (Mente). Madrid: Mestas Ediciones.

Asociación Española para la Calidad. (2013). Salario Emocional. Recuperado de https://www.aec.es/web/guest/centro-conocimiento/salario-emocional

Castillo, S. C. (2009). Nuevas tendencias en la retención y mejora del talento profesional y directivo. Recuperado de https://books.google.com.pe/books?id=Vy31Ph9gMX0C\&printsec=frontcover\&dq=s alario+emocional+segun+autores\&hl=es\&sa=X\&ved=0ahUKEwjgxNuglsTgAhXkhO AKHb1yAiIQ6AEIWjAJ\#v=onepage \&q\&f=false

Deloitte. (2018). Gestión de RRHH en América Latina Compensaciones salariales, perfiles y retos del área en la Región. Recuperado de https://www.coursehero.com/file/33946016/Gestion-de-RRHH-en-AmericaLatinapdf/

Mercer. (2018). Estudios 2018 sobre las Tendencias Globales de Talento. Impulsando el Potencial de Crecimiento en la Era Humana. Recuperado de http://www.mercermarshbeneficios.com.do/content/dam/marsh/Documents/PDF/LAC /LAC-es/Tendencias_Globales_de_Talento_Latam_2018\%20(2).pdf.

Sánchez, M. D. (2014). Comunicación efectiva y trabajo en equipo. Madrid: Editorial CEP Valls. J. (2017). Big Data: atrapando al consumidor. Barcelona: Profit Editorial

Vitteri, P. (20 de Abril de 2017). La Batalla por el Salario Emocional. Semana Económica. Recuperado de http://semanaeconomica.com/de-la-actitud-alexito/2017/04/20/salario-emocional/ 


\section{APÉNDICES}




\title{
APÉNDICE 1: CUESTIONARIO DE SALARIO EMOCIONAL
}

\author{
Cuestionario del Salario Emocional
}

El presente cuestionario tiene como propósito evaluar y determinar si dentro de la consultora de Recursos Humanos hay presencia o ausencia de salario emociona y tomar las medidas necesarias para poder realizar la implementación del mismo para el beneficio de la organización y colaboradores. El presente instrumento cuenta con 15 ítems los mismos que deber ser respondidos en su totalidad seleccionando solo una alternativa de respuesta.

1. Según su criterio la empresa proporciona regularmente normativas a seguir y planes de entrega de servicios que favorecen su crecimiento personal y organizacional.
A. NUNCA
B. POCAS OCACIONES
C. CASI SIEMPRE
D. SIEMPRE

2. Usted considera que la remuneración recibida es la justa y va acorde su trabajo.
A. NUNCA
B. POCAS OCACIONES
C. CASI SIEMPRE
D. SIEMPRE

3. Según su criterio la comodidad de su lugar de trabajo que le brinda la empresa satisface sus expectativas.
A. NUNCA
B. POCAS OCACIONES
C. CASI SIEMPRE
D. SIEMPRE

4. Considera que las opiniones que brinda son valoradas.
A. NUNCA
B. POCAS OCACIONES
C. CASI SIEMPRE
D. SIEMPRE

5. La empresa le ofrece la alternativa de trabajar en su casa de forma remota.
A. NUNCA
B. POCAS OCACIONES
C. CASI SIEMPRE 
D. SIEMPRE

6. La empresa le da facilidades para asistir a evaluaciones médicas o chequeos de rutina.
A. NUNCA
B. POCAS OCACIONES
C. CASI SIEMPRE
D. SIEMPRE

7. La flexibilidad de tiempos de entrega y productividad en la empresa es adecuado.
A. NUNCA
B. POCAS OCACIONES
C. CASI SIEMPRE
D. SIEMPRE

8. Considera que la organización se enfoca en satisfacer sus expectativas de motivación.
A. NUNCA
B. POCAS OCACIONES
C. CASI SIEMPRE
D. SIEMPRE

9. La empresa le da facilidades para poder equilibrar su vida personal y laboral (reuniones del colegio de sus hijos, cursos, talleres, etc.).
A. NUNCA
B. POCAS OCACIONES
C. CASI SIEMPRE
D. SIEMPRE

10. La empresa le otorga capacitaciones.
A. NUNCA
B. POCAS OCACIONES
C. CASI SIEMPRE
D. SIEMPRE

11. La organización le brinda algún tipo de reconocimiento por sobrepasar sus objetivos.
A. NUNCA
B. POCAS OCACIONES
C. CASI SIEMPRE 
D. SIEMPRE

12. La empresa le brinda facilidades para manejar sus horarios.
A. NUNCA
B. POCAS OCACIONES
C. CASI SIEMPRE
D. SIEMPRE

13. Recibe feedback de sus compañeros y jefes inmediatos.
A. NUNCA
B. POCAS OCACIONES
C. CASI SIEMPRE
D. SIEMPRE

14. Considera que la organización le permite explotar su potencial.
A. NUNCA
B. POCAS OCACIONES
C. CASI SIEMPRE
D. SIEMPRE

15. Se encuentra satisfecho con los beneficios que la empresa le brinda.
A. NUNCA
B. POCAS OCACIONES
C. CASI SIEMPRE
D. SIEMPRE 


\section{APÉNDICE 2: GUÍA DE ENTREVISTA}

1. ¿Cuánto tiempo tienes dentro de la consultora?

2. ¿Qué beneficios tienes dentro de la empresa?

3. ¿Cuál de estos beneficios crees que no encontrarías fácilmente en otra organización?

4. ¿Cuál es el beneficio más importante que tienes actualmente?

5. ¿Consideras que la organización tiene como parte de su cultura el concepto de flexibilidad?

6. ¿Te encuentras conforme con tu sueldo?

7. ¿La empresa te permita mantener un equilibrio entre lo personal y profesional?

8. ¿Qué otros beneficios te gustaría que la empresa te brinde?

9. ¿Por qué motivo buscarías otras ofertas laborales y no te quedarías en la empresa?

10. ¿Te encuentras actualmente en búsqueda de nuevas oportunidades laborales?

11. ¿Conoces el concepto de salario emocional? 